\title{
ARTIFICIAL INTELLIGENCE IN MEDICAL PRACTICE: REGULATIVE ISSUES AND PERSPECTIVES
}

DOI: 10.36740/WLek202012204

\author{
Vitalii M. Pashkov' ${ }^{1}$, Andrii 0. Harkusha ${ }^{2}$, Yevheniia 0. Harkusha ${ }^{2}$ \\ 'POLTAVA LAW INSTITUTE OF YAROSLAV MUDRYI NATIONAL LAW UNIVERSITY, POLTAVA, UKRAINE \\ ¿2ABORATORY FOR THE STUDY OF NATIONAL SECURITY PROBLEMS IN THE FIELD OF PUBLIC HEALTH OF ACADEMICIAN STASHIS SCIENTIFIC RESEARCH \\ INSTITUTE FOR THE STUDY OF CRIME PROBLEMS, KHARKIV, UKRAINE
}

\begin{abstract}
The aim of the research is to identify specific of Al in healthcare, its nature, and specifics and to establish complexities of Al implementation in healthcare and to propose ways to eliminate them.

Materials and methods: This study was conducted during June-0ctober of 2020. Through a broad literature review, analysis of EU, USA regulation acts, scientific researches and opinions of progressive-minded people in this sphere this paper provide a guide to understanding the essence of Al in healthcare and specifics of its regulation. It is based on dialectical, comparative, analytic, synthetic and comprehensive methods.

Results: One of the first broad definitions of Al sounded like "Artificial Intelligence is the study of ideas which enable computers to do the things that make people seem intelligent ... The central goals of Artificial Intelligence are to make computers more useful and to understand the principles which make intelligence possible." There are two approaches to name this technology - "Artificial intelligence" and "Augmented Intelligence." We prefer to use a more common category of "Artificial intelligence" rather than "Augmented Intelligence" because the last one, from our point of view, leaves much space for "human supervision" meaning, and that will limit the sense of Al while it will undoubtedly develop in future.

Al in current practice is interpreted in three forms, they are: Al as a simple electronic tool without any level of autonomy (like electronic assistant, "calculator"), Al as an entity with some level of autonomy, but under human control, and Al as an entity with broad autonomy, substituting human's activity wholly or partly, and we have to admit that the first one cannot be considered as Al at all in current conditions of science development. Description of Al often tends to operate with big technological products like DeepMind (by Google), Watson Health (by IBM), Healthcare's Edison (by General Electric), but in fact, a lot of smaller technologies also use Al in the healthcare field - smartphone applications, wearable health devices and other examples of the Internet of Things.

At the current stage of development Al in medical practice is existing in three technical forms: software, hardware, and mixed forms using three main scientific-statistical approaches - flowchart method, database method, and decision-making method. All of them are useable, but they are differently suiting for Al implementation. The main issues of Al implementation in healthcare are connected with the nature of technology in itself, complexities of legal support in terms of safety and efficiency, privacy, ethical and liability concerns.

Conclusion: The conducted analysis makes it possible to admit a number of pros and cons in the field of Al using in healthcare. Undoubtedly this is a promising area with a lot of gaps and grey zones to fill in. Furthermore, the main challenge is not on technology itself, which is rapidly growing, evolving, and uncovering new areas of its use, but rather on the legal framework that is clearly lacking appropriate regulations and some political, ethical, and financial transformations. Thus, the core questions regarding is this technology by its nature is suitable for healthcare at all? Is the current legislative framework looking appropriate to regulate Al in terms of safety, efficiency, premarket, and postmarked monitoring? How the model of liability with connection to Al technology using in healthcare should be constructed? How to ensure privacy without the restriction of Al technology use? Should intellectual privacy rights prevail over public health concerns? Many questions to address in order to move in line with technology development and to get the benefits of its practical implementation.
\end{abstract}

KEY WORDS: Al, Artificial Intelligence, Healthcare, Medical devices, Software

Wiad Lek. 2020;73(12 p. II):2722-2727

\section{INTRODUCTION}

Healthcare is one of the most rapidly growing spheres of sciences, so it is wide open for new technologies. One such being on the cutting edge is Artificial Intelligence (hereinafter - AI), which is started from entertainment and now spreading to other social life segments.

The trend of AI emerging is supported by several factors of modern life. Among them are the shortage of qualified doctors in the USA and Europe on the background of pop- ulation aging and the necessity of cost-effective treatment. According to prognoses [1], by 2050, in Europe and North America, one in four people will be the age of $65+$, and consequently, it will overload the healthcare system with aged patients with complex needs, long-term care management plan, and costly treatment. All that will demand switching of healthcare paradigm to meet new demands. So, there will be not only a need to attract and train more healthcare professionals increasing their number, but we 
also redistribute their workload focusing on patients' care avoiding time-spending on things that could and, in fact should be automated. And this scope is one of where AI has a massive potential to grow, modify healthcare, and address some ongoing and perspective challenges.

\section{THE AIM}

The research aims to identify specific of AI in healthcare, its nature, and peculiarities, to establish complexities of $\mathrm{AI}$ implementation in healthcare and to propose ways to eliminate them.

\section{MATERIALS AND METHODS}

This study was conducted during June-October of 2020. Through a broad literature review, analysis of EU, USA regulation acts, scientific researches and opinions of progressive-minded people in this sphere this paper provide a guide to understanding the essence of $\mathrm{AI}$ in healthcare and specifics of its regulation. It is based on dialectical, comparative, analytic, synthetic and comprehensive methods.

\section{RESULTS AND DISCUSSION}

In the earlier 1970s' "the possibility that the computer as an intellectual tool can reshape the present system of health care, fundamentally alter the role of the physician, and profoundly change the nature of medical manpower recruitment and medical education--in short, the possibility that the healthcare system by the year 2000 will be basically different from what it is today." [2] Countries like Finland, Germany, the UK, Israel, China, and the United States are intensively investing in AI-related research, and the dynamics of healthcare AI growth are unstable [3]: the USA still a "quantitive champion" with the biggest list of entities with the highest capitalization and broadest trials and researches, China is the one with the highest growth rate in healthcare AI implementation and intensive consumer-oriented approach (for example, Ping An Good Doctor) [4], European countries has advantages in terms of the scope of collected healthcare data and amount of joint researches in different issues of AI using in medicine such as data protection, privacy, ethics vs law, humanity and other. So, there is no single "flagship" till now, and the process of healthcare AI usage is at its starting point.

Going down to definition (its acronym - AI), we almost from the beginning meet some complexities. One of the first broad definitions sounded like "Artificial Intelligence is the study of ideas which enable computers to do the things that make people seem intelligent ... The central goals of Artificial Intelligence are to make computers more useful and to understand the principles which make intelligence possible." [5]. Now we have some "governmental," normative definitions. Thus, European-oriented terminology included in the European Commission's guidance on ethical AI as follows: "Artificial intelligence (AI) systems are software (and possibly also hardware) systems designed by humans that, given a complex goal, act in the physical or digital dimension by perceiving their environment through data acquisition, interpreting the collected structured or unstructured data, reasoning on the knowledge, or processing the information, derived from this data and deciding the best action(s) to take to achieve the given goal. AI systems can either use symbolic rules or learn a numeric model, and they can also adapt their behavior by analyzing how the environment is affected by their previous actions. [6] However, USA's approach is operating definition of "augmented intelligence," making an accent on the enhanced capabilities of human clinical decision making when coupled with these computational methods and systems." [7] Both approaches have their advantages and level of rationality, but the main two things we should consider are:

1) that $\mathrm{AI}$ in current practice is interpreted in three forms, they are: AI as a simple electronic tool without any level of autonomy (like electronic assistant, "calculator"), AI as an entity with some level of autonomy, but under human control, and AI as an entity with broad autonomy, substituting human's activity wholly or partly, and we have to admit that the first one cannot be considered as $\mathrm{AI}$ at all in current conditions of science development; 2) description of AI often tends to operate with big technological products like DeepMind (by Google), Watson Health (by IBM), Healthcare's Edison (by General Electric), but in fact, a lot of smaller technologies also use AI in healthcare field - smartphone applications, wearable health devices and other examples of the Internet of Things. We all (the majority of us) are using some assistants with AI technologies inside, and this trend is growing.

We prefer to use a more common category of "Artificial intelligence" rather than "Augmented Intelligence" because the last one, from our point of view, leaves a lot of space for "human supervision" meaning, and that will limit the sense of AI while it will undoubtedly develop in future. So, what is AI in medicine? Simply the AI specialized to medical applications.

At the current stage of development AI in medical practice is existing in three technical forms: software, hardware, and mixed forms.

Software form includes a wide range of implementations in applications from simply fixating and record-keeping to neural network systems designed to generalize data and recommend treatment decisions by predicting their efficiency. Most of its software-based potential AI in healthcare could demonstrate in the following areas: Artificial Intelligence Techniques in Medicine; Data Mining and Knowledge Discovery in Medicine; Medical Expert Systems; Machine Learning-Based Medical Systems; Medical Signal and Image Processing Techniques.

Hardware form is a "world of robotics" [8] that assists in medical treatment, surgeries, rehabilitation, functioning in intelligent prostheses, etc.

The mixed form is a combination of both elements as components of the complex unite system. For this moment, 
such a category is not filled with a lot of examples, in fact, we have just a few "almost-proper" examples of such [9].

Health care is faced with two modern problems that could be addressed by using AI, they are the rise of "big data" - huge amounts of data coming from many sources (electronic health records, scientific and practical medical literature, clinical trials and their results, insurance data, pharmacy records, information added by patients via smartphones, wearable devices etc.), and the necessity (and ability) to generalize and to find consistent patterns to enhance healthcare and treatment of patients. These two problems are resulting in one - the necessity of automatization and assistance, and it is curious, but healthcare system, thought being open to technologies, is one of the spheres with the lowest possible automatization rates - only $15 \%$ of working hours presumed to be automated till 2030 and only $35 \%$ - are potentially automatable at all [10]. Some skepticism is added by evidence-based researches arguably state that "... healthcare has exhibited a dismal record for adopting cutting edge technologies." [11]

How could AI help with this? The primary method of medical science is to establish interconnections through some kind of patterns based on existing data (databases), and here we can presume that statistical method was the core of such a process for a long time before AI. However, AI could be much more effective in that by using three main scientific-statistical approaches - flowchart method, database method, and decision-making method. All of them are useable but they are differently suiting for AI implementation.

"Flowchart method" is grounded on the gathering of established symptoms, thus creating some history record and resulting in probable diagnosis by combining symptoms in one picture. The downsides of such an approach are obvious, they are the necessity to input a lot of data on different symptoms, their characteristics, combinations, connected diseases etc. in order to achieve a trustworthy result; moreover, such an approach is limited because of medical worker's intermediary role - the algorithm cannot "ask" anything in addition except the information provided by the medical worker, cannot achieve any information of the particular patient - 100\% "machinery" and inflexible approach. However, it can be useful in appropriate circumstances, for instance, to encode triage protocols for use by nurses [12], patient interviewing [13], for giving therapeutic advice in the acid/base area [14].

"Database method" is based on the principle of self-generalization, self-learning, and in-depth analysis, when AI has to learn how to recognize interconnections and patterns utilizing repetitive algorithm designed to identify how the symptoms or their combinations, visual appearance etc. are manifested. And such systems are effectively working now, for instance, in the relevant issue of COVID-19 diagnostics on the basis of cough sound [15]. Although such data processing techniques have more advance than previous, it could not be implemented in all cases because of some issues: high costs and time spendings of collecting and processing huge databases; problems with the comparison of old data and new data; regional differences of collected data; the possibility of medical exceptions in some types of diseases; lack of explanation and inability to substitute the role of the physician. Such a method can undoubtedly be practically implemented, but the scope of such implementation should not be overestimated.

"Decision-making method" is grounded on the mathematical algorithms of creating decisions under some level of uncertainty, involving prior experience, manifestations, likelihoodness, and outcomes. As P. Szolovits rightly notes, "Besides its rationality, such an approach has some issues regarding obtaining reasonable estimates of probabilities and utilities for a particular analysis. Although techniques such as sensitivity analysis help greatly to indicate which potential inaccuracies are unimportant, the lack of adequate data often forces artificial simplifications of the problem and lowers confidence in the outcome of the analysis". [16] For instance, such an approach could lead to a situation when multiple symptoms are considering as a mix of single diseases (when, in fact, they are a combination leading to one) or vice versa. Additionally, numeric algorithmic representation of the decision-making process obviously differs from that of a real human-physician, which could confuse the patient.

Thus, every single approach is suitable but not universal, moreover, as was mentioned before, despite the fact of the relatively long story of AI, we can admit that it is just a starting point of the technology in general and its use in healthcare in particular. According to recent researches, "While there are widespread questions on what is real in AI in healthcare today, this report looked at 23 applications in use today and provides case studies of 14 applications already in use. These illustrate the full range of areas where AI can have an impact: from apps that help patients manage their care themselves, to online symptom checkers and e-triage AI tools, to virtual agents that can carry out tasks in hospitals, to a bionic pancreas to help patients with diabetes." [3, p. 14]

It is predicted that we will face three main phases of AI in healthcare scaling:

low-level technical implementation phase, when AI will be assisting in repetitive administrative tasks. At this point, $\mathrm{AI}$ technology will reduce the accompanying workload (not the main one) of the medical staff of all levels and image-based application of AI in ophthalmology, radiology [17].

home-based care phase, when the assistance of AI will make it possible to switch the model of medical treatment more toward remote monitoring, alerting visual assistance on the basis of AI technology. Additionally, advancing will take place in AI utilization in oncology, cardiology, neurology, where it shows its first forms of implementation these days [18-20] with broader digitalization combinations (by deep learning, NLP, connectivity) and organizational transformation in accompanying existing technologies.

clinical trials and decision support phase, when AI technologies will be implemented in clinical decision 
support, embedded in every stage of the healthcare system, from training and learning through clinical trials and treatment to health enhancement end general evaluation of healthcare

Thus, for now, we are far from the real broad implementation of AI in healthcare, so it is the right time to think - what kind of issues will it raise? Are we ready to address them, or (if not) what strategy should we have to minimize the risks? Furthermore, the central concern is grounded on the fact that we are discussing revolutionary modification of healthcare, public health issues, the conflict between public and private interests, law and ethics, technology, and humanity. We have no intention to dive into every single issue of AI implementation in healthcare deeply, and that will be a core of our further researches. So, let's get down to the analysis.

The technology nature. AI, machine learning, and supportive technologies, as we stated above, if we explain them with regard to healthcare, execute the process of obtaining the decision which usually is 1) unexplainable at all, because some of the stages are "presumed" or "skipped"; 2) explainable, but the explanation is justified mechanically but absolutely make no sense from a medical point of view [21]. That is why the term "black box" is widely used when describing the decision-making process within AI technology because the original algorithm could experience modifications on the basis of a massive amount of data analyzed or with changes of data over time. That could be positive when we analyze the perspective of image recognition and early prediction of, for example, skin cancer disease [22], identify disorders in infants on the basis of facial features [23], to recommend of-label use for existing drugs, etc. But medical science and medical treatment must be based on an appropriate level of certainty, so it would be a tough challenge to ensure effectiveness and safety and not interrupt progress, development, and use of AI technology benefits.

Law regulation approach. This issue's core is to ensure the quality, safety, and reliability of IA in healthcare. Regarding the primary status, such bodies as Food and Drug Administration in the USA, relevant Commission in the EU designed to oversee medical devices, but could AI - free-standing algorithms used to make medical decisions (or help make them) - be simply identified as regular medical device? [24, 25] They have many common characteristics, but legislative terms should be modified to strictly cover AI in all its forms. The question of efficiency and safety regarding medical objects always connected with two basic points of scientific reasoning plus understanding and approved by trial efficiency. And with both these demands, there are enormous complexities because understanding and reasoning are unattainable due to the essence ("black-box") of technology, and trials in their classical meaning may not be suitable to AI if the predicted results of AI algorithm isn't general but rather individual and personalized. Furthermore, one of the core features of AI using in healthcare is saving time to achieve the result, thus - trials will interrupt this feature majorly. Thus, the classical approach for premarket evaluation will hardly work for AI in healthcare, and broad premarket control should be changed to stricter and more comprehensive aftermarket monitoring to manage this challenge and to use a collaboration of various entities to create an AI algorithm of the highest quality.

Liability. Healthcare is a field where one can hardly underestimate the meaning of liability because a patient's health and life are at the center of this scope. Using some hiend technologies in medical practice to enhance treatment is not new, and this kind of model presumes that medical staff is professionally responsible for patient's care and all technical measures used. However, such a strict scheme will not (and in some circumstances should not) work that way when we are talking about AI if, for example, some issues of AI's algorithm caused a mistake in prioritization of patients by the urgency of care or mislead medical worker in some automatically measured figures of patient's state, etc. If AI is a simple electronic instrument under a physician's full supervision and control, it will be understandable case, but with the further increase of automated decision making, there will be an obvious need to clarify the borders and defining where professional responsibility begins and ends. We can now use the starting point of similarization of AI-related liability with the one connected to medical devices, where approach for regulating devices created by national bodies (FDA in USA, Medicines and Healthcare Products Regulatory Agency in UK, etc.). In this situation, the burden of assurance of device effectiveness and safety is fully on the regulatory body, and thus if an AI is used correctly, the physician must not be subject to liability. However, transforming AI from the concept of "supporting the decision" to "making the decision" is a matter of time, and then - should physicians overlook the results of AI activity and be liable for that? If yes - then it will decrease the attractiveness of technology in healthcare because of unnecessary "double-work", if no - how to ensure patient's rights in the right way? Should hospitals, physicians verify and test AI themselves before implementation? What should they do to fulfill their duties in using AI not to become liable? Without clarifying these questions, many physicians and healthcare organizations would be reluctant to introduce or significantly scale up AI applications in healthcare [3]. Liability issues are also connected with the concept of informed consent of the patient regarding treatment using AI technology, namely - how deep should be information that is delivered to the patient regarding AI and (because we a talking about very complex technology) will that information make it possible to such consent be really "informed" in fact.

Privacy. It is a trend of the 21 st century, and we cannot overlook this issue regarding AI use in healthcare, and three types of data processing are particularly relevant in this regard: collecting, analysis, and sharing. This data must be firstly loaded by the developers to "train" the AI, and then this data and its combined, generalized, sorted, evaluated, etc., forms are shared with other systems in order to exercise healthcare functions. But such kind of 
data is included in the category of highly sensitive data and is covered by special restrictive provisions of General Data Protection Regulation [26], Health Insurance Portability and Accountability Act's (HIPAA's) [27], and other acts and restriction of such information use need further clarification in terms of covered subjects, anonymization and other because machine learning and AI in general do not fully comply with current provisions on privacy. How could be addressed the situation with the right of a person to erase his/her data, if such data is already used, reorganized and "integrated" in a wide range of databases and influenced AI's algorithm and "decision-making"?

There are many other concerns of different nature, from intellectual property rights and commercialization of technology to ethical issues, unemployment concerns and conflict between public and private interests. We will try to address them in ongoing and further researches in this field.

\section{CONCLUSIONS}

The conducted analysis makes it possible to admit many pros and cons in the field of AI using in healthcare. Undoubtedly this is a promising area with a lot of gaps and grey zones to fill in. Furthermore, the main challenge is not on the technology itself, which is rapidly growing, evolving, and uncovering new areas of its use, but rather on the legal framework that is clearly lacking appropriate regulations and some political, ethical, and financial transformations. Thus, the core questions regarding this are as follows: is technology by its nature suitable for healthcare at all? Is the current legislative framework looking appropriate to regulate $\mathrm{AI}$ in terms of safety, efficiency, premarket, and postmarked monitoring? How the model of liability with connection to AI technology using in healthcare should be constructed? How to ensure privacy without the restriction of AI technology use? Should intellectual property rights prevail over public health concerns? Many questions to address in order to move in line with technology development and to get the benefits of its practical implementation.

\section{REFERENCES}

1. WHO report on aging. Available from: https://www.who.int/healthtopics/ageing\#tab=tab_1 [reviewed 2020.08.18].

2. Schwartz,W.B.,"Medicine and the Computer:The Promise and Problems of Change," New Engl. J. Med. 283,1970:1257-1264.

3. Transforming healthcare with AI The impact on the workforce and organizations March 2020. Available from: https://eithealth.eu/wpcontent/uploads/2020/03/EIT-Health-and-McKinsey_TransformingHealthcare-with-Al.pdf [reviewed 2020.08.18].

4. Ping An Good Doctor. Available from: https://en.wikipedia.org/wiki/ Ping_An_Good_Doctor [reviewed 2020.08.18].

5. Winston, P.W., Artificial Intelligence. Addison-Wesley, Reading, Mass. 1977.

6. European Commission, ethics guidelines for trustworthy Al 362019.

7. Am. Med. Assoc., Augmented Intelligence in Health Care, 2018 at 2. Available from: https://www.amaassn.org/system/files/2019-01/ augmented-intelligence-policy-report.pdf. [reviewed 2020.08.18].

8. Hamlet $P$, Tremblay J. Artificial intelligence in medicine. Metabolism. 2017; 69S:S36-40. [PubMed: 28126242].
9. Anthony Cuthbertson, Plug Pulled on Robot Doctor after Humans Complain, NEWSWEEK (Mar. 30, 2016), Available from: http:// www.newsweek.com/plug-pulled-robot-doctor-after-humanscomplain-442036 [reviewed 2020.08.05].

10. The future of work in Europe Automation, workforce transitions, and the shifting geography of employment: Available from: https://www. mckinsey.com/ /media/McKinsey/Featured\%20Insights/Future\%20 of\% 200 rganizations/The\%20future\%20of\%20work\%20in\%20Europe/ MGI-The-future-of-work-in-Europe-discussion-paper.pdf [reviewed 2020.08.10].

11. Nicolas Terry, Information Technology's Failure to Disrupt Healthcare, 13 NEV. L.J. 722 (2013).

12. Perlman, F., McCue, J.D., and Friedland, G., Urinary Tract Infection (UTI) / Vaginitis Protocol, Introduction. Ambulatory Care Project, Lincoln Laboratory, Massachusetts Institute of Technology. and Beth Israel Hospital, Harvard Medical School, (July 1974).

13. Slack, W. V., Van Cura, L. J., "Patient Reaction to Computer-Based Medical Interviewing," Comput. Biomed Res 1, 1968:527-531.

14. Bleich, H. L.,"Computer-Based Consultation: Electrolyte and Acid-Base Disorders," Amer. J. Med. 53, 1972:285.

15. COVID-19 Artificial Intelligence Diagnosis using only Cough Recordings Jordi Laguarta, Ferran Hueto, and Brian Subirana. Available from: https:// ieeexplore.ieee. $\mathrm{org} / \mathrm{stamp} / \mathrm{stamp}$.jsp?tp=\&arnumber $=9208795$ [reviewed 2020.08.21].

16. Szolovits, P. (Ed.). Artificial Intelligence in Medicine. Westview Press, Boulder, Colorado. 1982.

17. Ivan Cruz-AcevesFernando Cervantes-sanchezFernando CervantessanchezMaría Susana Avila GarcíaMaría Susana Avila García. Available from: https://www.researchgate.net/publication/324633520_A_ Novel_Multiscale_Gaussian-Matched_Filter_Using_Neural_ Networks_for_the_Segmentation_of_X-Ray_Coronary_Angiograms [reviewed 2020.08.21].

18. Halcox JPJ, Wareham K, Cardew A, Gilmore M, Barry JP, Phillips C, et al. Assessment of remote heart rhythm sampling using the AliveCor heart monitor to screen for atrial fibrillation: the REHEARSE-AF study. Circulation. (2017) 136:1784-94. doi: 10.1161/ CIRCULATIONAHA.117.030583.

19. Turakhia MP, Desai M, Hedlin H, Rajmane A, Talati N, Ferris T, et al. Rationale and design of a large-scale, app-based study to identify cardiac arrhythmias using a smartwatch: the apple heart study. Ame Heart J. (2019) 207:66-75. doi: 10.1016/j.ahj.2018.09.002.

20. Raja JM, Elsakr C, Roman S, Cave B, Pour-Ghaz I,Nanda A, et al. Apple watch, wearables, and heart rhythm: where do we stand? Ann Trans Med. (2019) 7:417. doi: 10.21037/atm.2019.06.79.

21. Jenna Burrell, How the Machine "Thinks": Understanding Opacity in Machine Learning Algorithms, 3 Big Data \& Soc'y 1, 52016.

22. Andre Esteva et al., Dermatologist-Level Classification of Skin Cancer with Deep Neural Networks, 542 Nature 1152017.

23. Megan Molteni, Thanks to Al, Computers Can Now See Your Health Problems, Wired (Jan. 9, 2017). Available from: https:// www.wired.com/2017/01/computers-can-tell-glance-youve-gotgeneticdisorders/. [reviewed 2020.08.21].

24. Vitalii Pashkov, Andrii Harkusha: Certain aspects on medical devices software law regulation. Wiadomości Lekarskie 2016;tom LXIX;nr 6: 765-767.

25. Vitalii Pashkov, Nataliya Gutorova, Andrii Harkusha /Medical device software: defining key terms// Wiadomości Lekarskie 2016;tom LXIX;nr 6:813-817. 
26. General Data Protection Regulation. Available from: https://gdpr-info. eu/ [reviewed 2020.08.21].

27. Health Insurance Portability and Accountability Act's. Available from: https://www.cdc.gov/phlp/publications/topic/hipaa.html [reviewed 2020.08.21].

\section{ORCID and contributions:}

Vitalii M. Pashkov: 0000-0001-9489-7768 A,B,D,E,F

Andrii O. Harkusha: 0000-0001-5266-3007 A,B,D,E,F

Yevheniia O. Harkusha: 0000-0002-9932-8756 ${ }^{A, B, D, E, F}$

\section{Conflict of interest:}

The Authors declare no conflict of interest.

\section{CORRESPONDING AUTHOR}

Vitalii M. Pashkov

Poltava Law Institute of Yaroslav Mudriy

National Law University, Poltava, Ukraine

tel: +38066 6931651

e-mail: v.pashkov26.06@ukr.net

Received: 01.09 .2020

Accepted: 30.11 .2020

A - Work concept and design, B - Data collection and analysis, C - Responsibility for statistical analysis,

D-Writing the article, $\mathbf{E}$-Critical review, $\mathbf{F}$ - Final approval of the article 